\title{
Identification of phenothiazine as an ETV1-targeting agent in gastrointestinal stromal tumors using the Connectivity Map
}

\author{
CHUEH-CHUAN YEN ${ }^{1,2}$, LI-TZONG CHEN ${ }^{3}$, CHIEN-FENG LI ${ }^{4}$, SAN-CHI CHEN ${ }^{1,2}$, WEI-YANG CHUA ${ }^{1}$, \\ YUNG-CHAN LIN ${ }^{1}$, CHIAO-HAN YEN ${ }^{1}$, YEN-CHUN CHEN ${ }^{1}$, MUH-HWA YANG $^{1,2}$, \\ YEE CHAO $^{1,2}$ and JONATHAN A. FLETCHER ${ }^{5}$
}

\begin{abstract}
${ }^{1}$ Division of Medical Oncology, Center for Immuno-oncology, Department of Oncology, Taipei Veterans General Hospital, Taipei 11217; ${ }^{2}$ National Yang-Ming University School of Medicine, Taipei 11221; ${ }^{3}$ National Institute of Cancer Research,

National Health Research Institutes, Tainan 70456; ${ }^{4}$ Department of Pathology, Chi-Mei Medical Center, Tainan 71004, Taiwan, R.O.C.; ${ }^{5}$ Department of Pathology, Brigham and Women's Hospital, Boston, MA 02115, USA
\end{abstract}

Received November 1, 2018; Accepted June 12, 2019

DOI: 10.3892/ijo.2019.4829

\begin{abstract}
Gastrointestinal stromal tumors (GISTs) are gastrointestinal tract sarcomas that commonly contain a mutation in the tyrosine kinases, KIT and platelet-derived growth factor receptor A (PDGFRA). Imatinib, sunitinib and regorafenib are all effective tyrosine kinase inhibitors; however, acquired resistance is inevitable. The E26 variant 1 (ETV1) pathway has been found to be a key downstream effector of KIT and is therefore a reasonable therapeutic target for this disease. In this study, we explored the potential agents targeting ETV1 in GISTs by uploading an ETV1 knockout gene signature of GIST cell lines to the pattern-matching software 'Connectivity Map'. The activity and mechanisms of identified agents were examined using an in vitro model. Four drugs were identified: Suberanilohydroxamic acid and trichostatin [two histone deacetylase inhibitors (HDACIs)] and trifluoperazine and thioridazine (two phenothiazine-class drugs). Western blot analysis demonstrated that all four drugs had ETV1-downregulating effects. As HDACIs have been previously studied in GISTs, we focused on phenothiazine. Phenothiazine was found to exert cytotoxicity and to induce apoptosis and autophagy in GISTs. Treatment with phenothiazine had little effect on the KIT/AKT/mammalian target of rapamycin (mTOR) pathway, but instead upregulated extracellular-signal-regulated kinase (ERK) activity. A combination of phenothiazine and a MEK inhibitor had a synergistic cytotoxic effect on GISTs. Western blot analysis
\end{abstract}

Correspondence to: Dr Chueh-Chuan Yen, Division of Medical Oncology, Center for Immuno-oncology, Department of Oncology, Taipei Veterans General Hospital, No. 201, Section 2, Shih-Pai Road, Taipei 11217, Taiwan, R.O.C.

E-mail: ccyen@vghtpe.gov.tw

Key words: Connectivity Map, E26 variant 1, gastrointestinal stromal tumors, phenothiazine indicated that ELK1 and early growth response 1 (EGR1) were activated/upregulated following phenothiazine treatment, and the MEK inhibitor/phenothiazine combination downregulated the ERK/ELK1/EGR1 pathway, resulting in diminished autophagy, as well as enhanced apoptosis. On the whole, the findings of this study established phenothiazine as a novel class of therapeutic agents in GIST treatment and demonstrate that a combination of phenothiazine and MEK inhibitor has great potential for use in the treatment of GISTs.

\section{Introduction}

Gastrointestinal stromal tumors (GISTs) are the most common mesenchymal tumors of the gastrointestinal tract, occurring most frequently in the stomach (approximately $50 \%)$ and small intestine $(25-35 \%)$ and less frequently in the colorectal regions (10-12\%), omentum/mesentery (7\%) and esophagus (1-5\%) (1-3). The majority of GISTs contain mutations in the gene encoding the KIT tyrosine kinase receptor (4); however, but $<10 \%$ have mutations in the gene encoding platelet-derived growth factor receptor A (PDGFRA) as the oncogenic driving force(5). Imatinib mesylate (Novartis Pharmaceuticals), a potent tyrosine kinase inhibitor (TKI) for both KIT and PDGFRA, is the standard first-line therapy and can achieve a median overall survival (OS) rate of 5-6 years in patients with advanced disease $(6,7)$. However, disease progression, which most likely occurs due to the development of secondary mutations $(8,9)$, is expected within 2 to 3 years of imatinib therapy $(6,7)$. Therapeutic options are limited for these patients, with sunitinib maleate (Pfizer) as the standard second-line agent (10), and regorafenib (Bayer Schering Pharma AG) as third-line therapy $(11,12)$.

KIT and its downstream pathway remain a major target of interest in TKI-refractory GISTs. Therapeutic strategies, such as those involving the degradation of the KIT protein [by using either a heat shock protein 90 inhibitor (13) or histone deacetylase inhibitors (14)] or the targeting of its downstream effectors by blocking phosphoinositide 3-kinase (15) or mammalian target of rapamycin (mTOR) (16), have been shown to be effective in inhibiting GIST growth. However, 
only a few of these have been successfully applied in clinical studies (17-19). Consequently, identifying novel therapeutic targets or strategies against GIST is a priority.

By using bioinformatics analysis, Chi et al discovered that ETS family member E26 variant 1 (ETV1) is highly expressed in GISTs (20). They also demonstrated that interstitial cell of Cajal (ICC) hyperplasia in mouse models expressing constitutively activated KIT also strongly expressedETV1. Their analysis revealed that genes that were downregulated by ETV1 knockdown demonstrated a negative correlation with genes that were upregulated in GISTs, suggesting a key role of ETV1 in an ICC-GIST-specific transcription network. Furthermore, their study demonstrated that the ETV1 transcriptional program is further regulated by activated KIT and its downstream MAP kinase, which prolongs ETV1 protein stability and cooperates with ETV1 to promote tumorigenesis (20). In their subsequent study, Ran et al uncovered a positive feedback circuit in which the KIT/MAP kinase pathway stabilized the ETV1 protein and ETV1 positively regulated KIT expression. The combined targeting of ETV1 stability by using imatinib and MEK162 resulted in significant in vitro and in vivo cytotoxic effects (21). This series of studies demonstrated that ETV1 is a lineage-specific oncogenic transcription factor required for the growth and survival of GISTs, and that the identification of novel agents that target the ETV1 pathway may be a strategy for the treatment of GISTs.

Lamb et al created a reference collection of gene expression profiles from cultured human cells treated with diverse bioactive small molecules, as well as pattern-matching software for data mining ['The Connectivity Map' (CMAP), http://www.broad.mit.edu/cmap/] $(22,23)$. When any gene expression profiles of interest are uploaded to this database, pattern-matching algorithms score each reference profile for the direction and strength of enrichment with the query signature. Perturbagens are then ranked by this 'connectivity score'; those at the top ('positive') and bottom ('negative') are functionally connected with the query state through the transitory feature of common gene-expression changes (22). In this study, an ETV1 knockout gene signature of GIST cell lines was uploaded, and phenothiazine was identified as a class of ETV1-targeting agent in GISTs.

\section{Materials and methods}

Collection of expression profiles from public datasets. Microarray and clinicopathological data from the Gene Expression Omnibus (GEO) datasets GSE14827 [osteosarcoma (OS)], GSE13433 [alveolar soft part sarcoma (ASPS)], GSE8167 (GIST), GSE20196 [synovial sarcoma (SynSA)], GSE20559 [liposarcoma (LPS)] and GSE17679 [Ewing sarcoma family tumors (ESFT)] were obtained from the NCBI website. E-MEXP-1922 [leiomyosarcoma (LMS)] data were obtained from the ArrayExpress website. All datasets were in the Affymetrix U133 Plus 2.0 platform. In order to reduce intra-subtype heterogeneity, we did not include all samples. For OS, only tumors without subsequent metastasis were selected; for GISTs, only those with exon 11 mutations were included; for SynSA, only tumors with SYT-SSX type 1 fusion gene and non-poorly differentiated histology were analyzed; and for LPS, only well- and de-differentiated tumors were chosen due to their similar genetic background. Expression profile data of GIST cell lines with ETV1 wild-type versus knockout were also obtained from GEO (GSE19396) (20).

Bioinformatics analysis. dChip $(24,25)$ was the main analytic software used, and the normalization of the expression values of individual chips was performed according to software specifications. One-way analysis of variance (ANOVA) was used to identify genes showing differentiated expression between different sarcoma subtypes, as well as different mutation status categories in GISTs. The expression levels of individual genes were obtained using z-score transformation, and the differences between different subtypes were then compared using the t-test.

Chip data from GSE19396 were analyzed separately. The dChip 'Compare Samples' function was used to explore differentially expressed genes between wild-type and ETV1 knockdown strains of GIST cell lines using 1.4-fold changes (with $\mathrm{P}<0.05$ by the unpaired t-test) as the threshold (24) (please also refer to https://sites.google.com/site/dchipsoft/high-level-analysis/compare-samples/comparison-criteria).

Gene signature query of CMAP. The identified gene signature was first transformed to probe set ID of Human Genome U133A. Up- and downregulated ID lists were then uploaded to the CMAP website (http://www.broad.mit.edu/cmap/). The query signature was then compared with each rank-ordered list to determine whether upregulated query genes tend to appear near the top of the list and downregulated query genes near the bottom ('positive connectivity'), or vice versa ('negative connectivity'), yielding a 'connectivity score' ranging from +1 to -1 . A null (zero) connectivity score was assigned where the enrichment scores for the up- and downregulated genes had the same sign. All instances in the database were then ranked according to their connectivity scores; those at the top were most strongly associated with the query signature, and those at the bottom were most strongly anti-associated $(22,23)$.

Cell lines and mass ARRAY-based mutation characterization. GIST cell lines were kindly provided by Dr J.A. Fletcher, and the KIT mutation status of this line has been previously described (13). GIST882 is a human cell line established from an untreated GIST with a primary imatinib-sensitive homozygous missense mutation in KIT exon 13, encoding a K642E mutant KIT oncoprotein (26). GIST48 was established from GISTs that had progressed, after initial clinical response, during imatinib therapy. GIST48 has a primary, homozygous exon 11 missense mutation (V560D) and a heterozygous secondary exon 17 (kinase activation loop) mutation (D820A) (13).

The sequences of KIT of both cell lines were surveyed again by Mass ARRAY-based mutation characterization before this study. The Sequenom MassARRAY platform utilizes a homogeneous reaction format with a single extension primer to generate allele-specific products with distinct masses $(27,28)$. PCR and extension primers for the mutations were designed using MassArray Assay Design 3.1 software (Sequenom). The mutation alleles were manually designed by extension in either the forward or reverse direction to have lower mass than the reference allele. The PCR products of multiplexed reactions were spotted onto SpectroCHIP II arrays, and DNA fragments were resolved by on the MassARRAY Analyzer 4 
System (Sequenom). Each spectrum was then analyzed using the Typer 4.0 software (Sequenom) to call mutations. Putative mutations were further filtered by manual review. The results of the mutation analysis of two cell lines are shown in Fig. S1, and their mutation status remained the same.

Reagents. The drugs used in this study were imatinib (ALX-270-492; Enzo Life Sciences), PD98059 (P215), suberanilohydroxamic acid (SAHA) (SML0061), trifluoperazine (TFP) (T8516), thioridazine (TDZ) (T9025) (all from Sigma-Aldrich) and MEK162 (S7007; Selleckchem). The primary antibodies used for western blot analysis included p-ERK (cat. no. 4376; 1:1,000), KIT (cat. no. 3392; 1:1,000), p-KIT (cat. no. 3391; 1:1,000), AKT (cat. no. 9272; 1:1,000), p-AKT (cat. no. 4058; 1:1,000), p-p70S6K (cat. no. 9205 ; 1:500), LC3 (cat. no. 2775; 1:1,000), early growth response protein 1 (EGR1) (cat. no. 4153; 1:1,000) and poly-(ADP-ribose) polymerase (PARP) (cat. no. 9542; 1:1,000) from Cell Signaling Technology; actin (cat. no. ab6276-100; 1:10,000) and ETV1 (cat. no. ab184120; 1:1,000) from Abcam Biotechnology; and ERK (cat. no. sc-135900; 1:1,000), ELK1 (cat. no. sc-355; 1:200) and p-ELK1 (cat. no. sc-8406; 1:200) from Santa Cruz Biotechnology. The secondary antibodies used were horse anti-mouse IgG (cat. no. 7076; 1:3,000) and goat anti-rabbit IgG (cat. no. 7074; 1:3,000), both from Cell Signaling Technology.

3-(4,5-Dimethylthiazol-2-yl)-2,5-diphenyltetrazolium bromide assay. Cell viability was measured using the TACS ${ }^{\mathrm{TM}}$ 3-(4,5-dimethylthiazol-2-yl)-2,5-diphenyltetrazolium bromide (MTT) cell proliferation assay (R\&D Systems) according to the manufacturer's instructions. Briefly, the cells were plated in 96-well plates at a concentration of 2,000-20,000/100 $\mu 1 /$ well overnight. Drugs at various concentrations were added in triplicate. The plates were incubated for the desired time at $37^{\circ} \mathrm{C}$, pulsed with $10 \mu \mathrm{l}$ of MTT reagent, and incubated for an additional $4 \mathrm{~h}$ at $37^{\circ} \mathrm{C}$. Detergent reagent at $200 \mu \mathrm{l} /$ well was added and mixed thoroughly to dissolve the dark blue crystals. The absorbance of the converted dye was measured spectrophotometrically in a Vmax microplate reader (Molecular Devices) at wavelengths of $570 \mathrm{~nm}$ (test) and $650 \mathrm{~nm}$. Cell survival was calculated as the percentage of MTT inhibition as follows: $\%$ survival $=($ mean experimental absorbance $/$ mean control absorbance) x100 (29).

The synergistic effect of the applied drug combination was measured through a combination index $(\mathrm{CI})$, which was calculated using CalcuSyn software (Biosoft) (30). CI $>1$ was defined as antagonism, $\mathrm{CI}=1$ as additivity, and $\mathrm{CI}<1$ as synergy; the experiment was performed in triplicate.

Western blot analysis. Monolayers of cultured cells were rinsed in phosphate-buffered saline (PBS) and scraped into lysis buffer [25 nM Tris. $\mathrm{HCl} \mathrm{pH} 7.6,150 \mathrm{nM} \mathrm{NaCl}$, $1 \%$ NP-40, $1 \%$ sodium deoxycholate, $0.1 \%$ SDS (Thermo Fisher Scientific)] containing the Protease and Phosphatase Inhibitor Cocktail (1:100 dilution; Thermo Fisher Scientific). Lysates were incubated for $30 \mathrm{~min}$ at $4^{\circ} \mathrm{C}$ and then clarified by centrifugation for $30 \mathrm{~min}$ at $13,200 \mathrm{rpm}$ at $4^{\circ} \mathrm{C}$. Protein concentrations were determined with the Pierce BCA Protein Assay kit (Thermo Fisher Scientific). Protein extracts
(20-50 $\mu \mathrm{g}$ per lane) were electrophoretically separated on sodium dodecyl sulfate-polyacrylamide gel electrophoresis gels (8-12\% depending on the molecular weights of proteins), transferred to polyvinylidene difluoride membranes (PerkinElmer), and blotted with specific antibodies. For primary antibodies, the sample was incubated at $4^{\circ} \mathrm{C}$ overnight; for secondary antibody, the sample was incubated at room temperature for $1 \mathrm{~h}$. Immunoreactive bands were detected using enhanced chemiluminescence (Millipore) and $\mathrm{X}$-ray film.

Measurement of caspase activity. Caspase activity was detected using the Caspase-Glo ${ }^{\circledR}$ 3/7 assay kit (Promega) according to the manufacturer's instructions. Briefly, GIST cells $\left(10^{4}\right.$ cells/well $)$ were seeded in a luminometer plate and incubated for $24 \mathrm{~h}$ at $37^{\circ} \mathrm{C}$. GIST cells were treated with DMSO (control vehicle) or with various concentrations of drugs for $72 \mathrm{~h}$. Moreover, $100 \mu \mathrm{l}$ of caspase-3/7 reagents was added to each well and mixed gently using a plate shaker. The cells were then incubated for 0.5-1 $\mathrm{h}$ at room temperature, and the luminescence of each well was measured.

Apoptosis assessment by Annexin V staining. The cells were plated at a density of 350,000 cells/well or 1,000,000 cells/well in 6-well plates. The following day, the cells were treated with TFP $(0,30$ and $40 \mu \mathrm{M})$ or TDZ $(0,25$ and $35 \mu \mathrm{M})$ for $72 \mathrm{~h}$, and then washed with 1X PBS for twice and resuspended in $100 \mu \mathrm{l}$ staining solution containing Annexin V-APC (BD Pharmingen) and propidium iodide in Annexin V-binding buffer. The cells were then incubated at room temperature for $15 \mathrm{~min}$, and the cells were then diluted in $400 \mu \mathrm{l} 1 \mathrm{X}$ Annexin V-binding buffer. The percentages of apoptotic cells were then measured using a flow cytometer (Canto II; BD Biosciences).

Detection and quantification of autophagic cells by staining with acridine orange. Cells were seeded in 6-well tissue culture dishes and treated with TFP (GIST882, $20 \mu \mathrm{M}$; GIST48, $10 \mu \mathrm{M}$ ) for $72 \mathrm{~h}$. The cells were then incubated at room temperature with medium containing acridine orange $(100 \mathrm{mg} / \mathrm{ml})$ for $15-20 \mathrm{~min}$, washed once with PBS, and fresh media were added. Fluorescent micrographs were then acquired using an inverted fluorescence microscope (IX51; Olympus). All images presented are at the same magnification (x200). The number of cells with increased acidic vesicular organelles was determined by flow cytometry. The cells were trypsinized and harvested. Red fluorescence emission was measured through a FACSCalibur from BD Biosciences using CellQuest software.

Statistical analysis. As stated above, dChip 'Compare Samples' function with 1.4-fold changes set as the threshold (with $\mathrm{P}<0.05$ by the unpaired t-test) was used to identify differentially expressed genes between control $(n=4)$ and ETV1 knockdown strains $(n=8)$ of GIST cell lines. The differences of expression level of individual genes between GISTs and non-GIST subtypes, and the difference of percentage of viability (assessed by MTT cell proliferation assay) and the percentage of apoptotic cells (assessed by apoptosis assay) between the control and drug treatment (all in triplicate), were compared using ANOVA, and the Bonferroni test was 

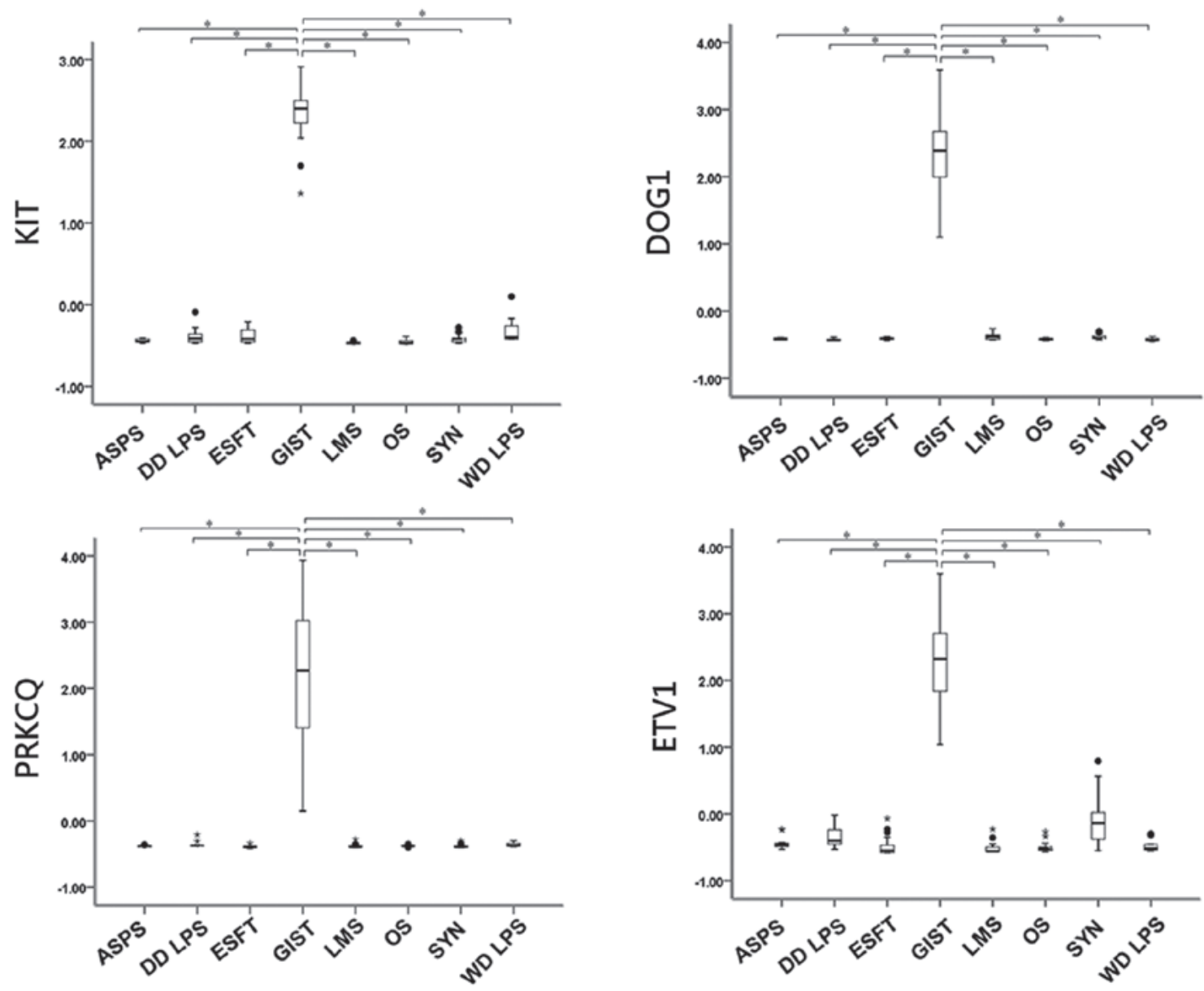

Figure 1. Differential expression of genes in GISTs and non-GIST sarcoma. The expression levels of individual genes were obtained using z-score transformation, and the differences between GISTs and non-GIST subtypes were then compared using the ANOVA (with Bonferroni test as a post hoc test). KIT, DOG1, PKCQ and ETV1 all showed significantly high expression levels in GISTs in comparison with other non-GIST sarcomas ("P<0.00001). GISTs, gastrointestinal stromal tumors; ASPS, alveolar soft part sarcoma; DD LPS, de-differentiated liposarcoma; ESFT, Ewing sarcoma family tumors; LMS, leiomyosarcoma; OS osteosarcoma; WD LPS, well-differentiated liposarcoma.

used as a post hoc test (with $\mathrm{P}<0.05$ considered to indicate a statistically significant difference).

\section{Results}

Confirmation of the overexpression of ETV1 in GIST in comparison with other sarcomas. Eight different types of sarcoma were analyzed: GIST, OS, ASPS, SynSA, well-differentiated LPS (WD LPS), de-differentiated LPS (DD LPS), ESFT and LMS. Comparisons of the expression levels of three well-known GIST-specific expression genes, namely KIT, DOG1 and PRKCQ and ETV1, were made. As shown in Fig. 1, KIT, DOG1, PRKCQ and ETV1 were all significantly highly expressed in the GISTs in comparison with the other sarcomas.

Identification of novel agents targeting the ETV1 pathway in GIST by CMAP. We downloaded the expression profile information from the GEO database (GSE19396) created by Chi et al (20) and compared the expression profiles of wild-type GISTs and ETV1-knockdown GISTs. We obtained a total of 207 probes of genes with differential expression between these two types of cell lines (Tables SI and SII). We then uploaded this set of genes onto CMAP to search for possible agents. The five drugs that had the most significant P-values following permutation are presented
Table I. Top 5 agents identified by CMAP after uploading ETV1 knockdown vs. wild-type expression profiles.

\begin{tabular}{llrl}
\hline Rank & \multicolumn{1}{c}{ CMAP name } & No. & P-value \\
\hline 1 & Prestwick-1080 & 4 & $<0.00001$ \\
2 & Suberanilohydroxamic acid & 12 & $<0.00001$ \\
3 & Trichostatin A & 182 & $<0.00001$ \\
4 & Trifluoperazine & 16 & $<0.00001$ \\
5 & Thioridazine & 20 & $<0.00001$ \\
\hline
\end{tabular}

CMAP, ‘The Connectivity Map'; ETV1, E26 variant 1.

in Table I. Prestwick-1080 is unavailable. SAHA and trichostatin are HDACIs, of which anti-GIST activity has been previously demonstrated (14). However, TFP and TDZ, two drugs of the phenothiazine class, have not been reported to date to have cytotoxic activity against GISTs, at least to the best of our knowledge. Therefore, we focused on these two drugs in this study. KIT-ETV1 in GIST cell lines. Subsequently, we examined 

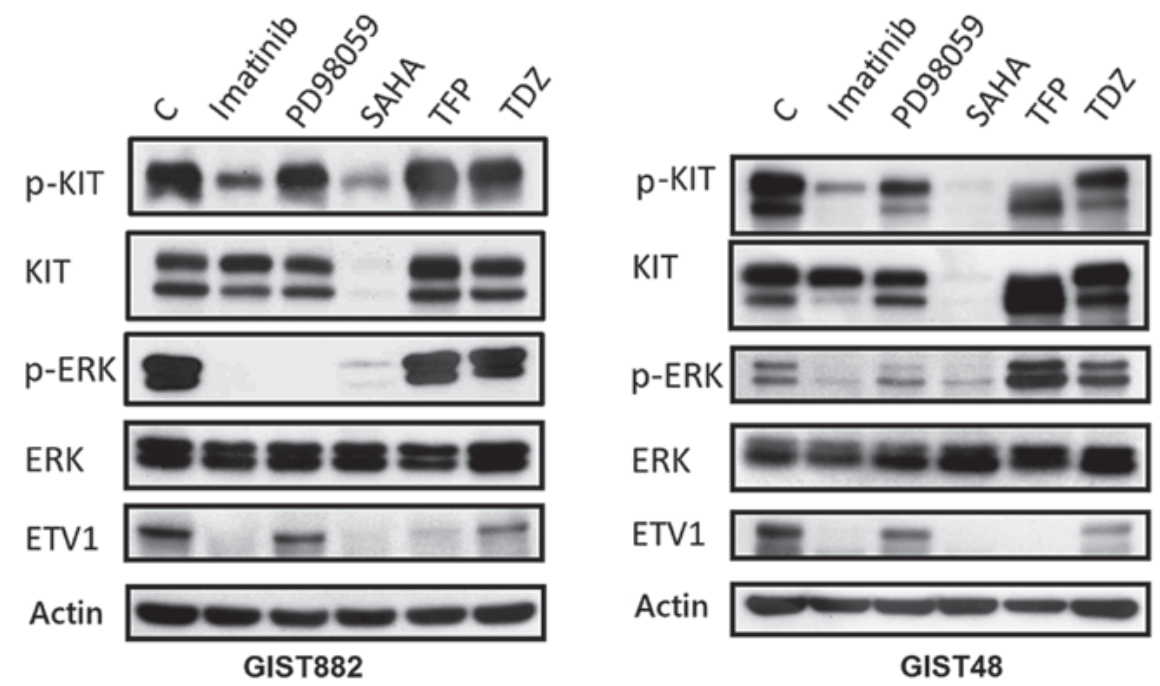

Figure 2. Western blot analysis of GISTS 882 and GIST48 cells following treatment with various agents. Imatinib $(2 \mu \mathrm{M}, 12 \mathrm{~h}$ for GIST882 and $20 \mu \mathrm{M}, 12 \mathrm{~h}$ for GIST48) inhibited KIT and downstream ERK, resulting in ETV1 downregulation. The MEK inhibitor, PD98059 (100 nM, 12 h), inhibited ERK with subsequent ETV1 downregulation. SAHA $(10 \mu \mathrm{M}, 12 \mathrm{~h})$, an HDACI, degraded KIT with the subsequent inhibition of ERK and the decreased expression of ETV1. TFP ( $35 \mu \mathrm{M}, 72 \mathrm{~h}$ for GIST882; $25 \mu \mathrm{M}, 72 \mathrm{~h}$ for GIST48) and TDZ ( $20 \mu \mathrm{M}, 72 \mathrm{~h}$ for GIST882; $12.5 \mu \mathrm{M}, 72 \mathrm{~h}$ for GIST48) downregulated ETV1, with little change in AKT and little change in, or even the paradoxical activation of, the ERK pathway. C, control; SAHA, suberanilohydroxamic acid; TFP, trifluoperazine; TDZ, thioridazine.

the targeting effect of TFP and TDZ, as well as other agents, on the KIT/ETV1 pathway. Representative western blots of GISTS882 and GIST48 following treatment with different agents are shown in Fig. 2. The inhibition of KIT signaling by imatinib $(2 \mu \mathrm{M}, 12 \mathrm{~h})$ in the imatinib-sensitive GIST882 cells resulted in MEK pathway inhibition, as well as in ETV1 protein downregulation. The imatinib-resistant GIST48 cells exhibited changes to relevant pathways following treatment with imatinib at a higher concentration $(20 \mu \mathrm{M}, 12 \mathrm{~h})$. By contrast, both GIST cell lines treated with the MEK inhibitor, PD98059 (100 nM, $12 \mathrm{~h})$, exhibited an inhibition of ERK phosphorylation and the loss of ETV1. Previous research has demonstrated that KIT activity and expression, and the activation of downstream pathways in GISTs are strongly inhibited by HDACI (14). In this study, we also found that SAHA (10 $\mu \mathrm{M}, 12 \mathrm{~h})$, an HDACI, degraded KIT, resulting in a subsequent decrease in ETV1 expression (Fig. 2).

We then further explored the targeting effects of TFP ( $35 \mu \mathrm{M}, 72 \mathrm{~h}$ for GIST882; $25 \mu \mathrm{M}, 72 \mathrm{~h}$ for GIST48) and TDZ $(20 \mu \mathrm{M}, 72 \mathrm{~h}$ for GIST882; $12.5 \mu \mathrm{M}, 72 \mathrm{~h}$ for GIST48) on GIST cell lines. As shown in Fig. 2, ETV1 in the GIST882 and GIST48 cells was downregulated by TFP and TDZ. However, the expression levels of both total and activated KIT were not markedly affected by these two drugs. This result confirms that both TFP and TDZ have distinct targeting effects on the ETV1 pathway, compared with other drugs.

Both TFP and TDZ exert cytotoxic effects against GISTs. We then evaluated the cytotoxic effects of TFP on GIST cell lines. As shown in Fig. 3A and B, both TFP and TDZ exerted significant anti-proliferative effects on the GIST48 and GIST 882 cells in a dose-dependent manner. However, TFP induced caspase activation in the GIST48 cells, but not in the GIST882 cells (Fig. 3C and D). A significant increase in the number of apoptotic cells, as assessed by Annexin V staining, was easily observed in the GIST48 cells treated with both agents. However, this could only be seen in the GIST882 cells at relatively higher concentrations of phenothiazine-class drugs (Fig. 3E and F). These results indicate that both TFP and TDZ exert a cytotoxic effect against GISTs, but not always through apoptosis.

TFP and TZD induce autophagy and the downregulation of ETV1 with the paradoxical upregulation of ERK in GIST cell lines. Phenothiazine drugs are a well-known class of drugs that induce autophagy (31). In this study, we evaluated the possibility of autophagy induction in GIST cells following drug treatment. As illustrated in Fig. 4, both TFP and TDZ induced autophagy in GISTs, as demonstrated by an increased LC3-II expression. Quantitative fluorescence-activated cell sorting (FACS) analysis revealed an increase in lysosomes following drug treatment (Fig. 5). These results indicate that TFP and TDZ can induce autophagy in GIST cell lines. PARP cleavage, an indicator of apoptosis, was also observed in the GIST48 and, to a lesser extent, in the GIST882 cells. This is compatible with the distinct pattern of caspase activation observed in both cell lines following treatment with TFP.

Chi et al revealed that ETV1 was a downstream effector of MEK and that MEK inhibition causes ETV1 downregulation (20). Similar findings were also noted in this study (Fig. 2). However, the GIST cell lines treated with TFP or TDZ exhibited an ETV1 downregulation with a paradoxical ERK activation (Fig. 4), indicating a MEK-independent mechanism in ETV1 degradation. TFP induced the suppression of the AKT (indicated by p-AKT)/mTOR (indicated by p-p70s6k) signaling pathway in the GIST48 cells at a relatively high concentration $(25 \mu \mathrm{M})$. In addition, the downregulation of p-KTI was observed in the TFP-treated GIST48 (20 and $25 \mu \mathrm{M})$ and GIST882 $(35 \mu \mathrm{M})$ cells. No marked changes in the KIT/AKT/mTOR signaling were observed in the TDZ-treated GIST cells. 

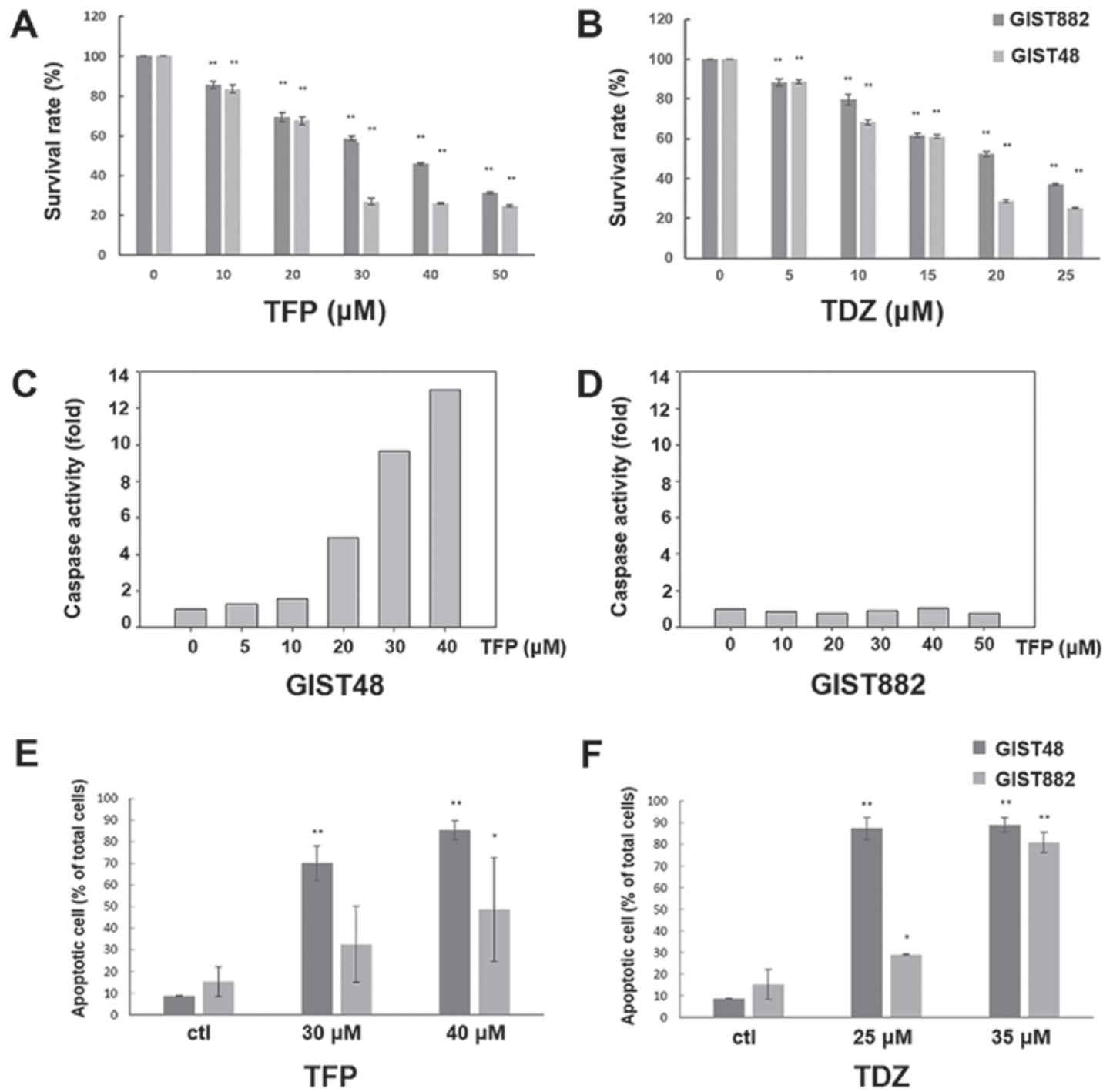

Figure 3. Evaluation of the viability and apoptosis of GIST882 and GIST48 cell lines following phenothiazine treatment: TACS ${ }^{\mathrm{TM}}$ MTT cell proliferation assay following treatment with (A) TFP and (B) TDZ for $72 \mathrm{~h}$; caspase activation assay of (C) GIST48 and (D) GIST882 following treatment with TFP; apoptosis assessment by Annexin V staining in GIST882 and GIST48 cell lines following treatment with (E) TFP or (F) TDZ for $72 \mathrm{~h}\left({ }^{*} \mathrm{P}<0.05,{ }^{* *} \mathrm{P}<0.01\right.$ in comparison with control using ANOVA, with Bonferroni test as a post hoc test). TFP, trifluoperazine; TDZ, thioridazine.

TFP or TDZ act synergistically with the MEK inhibitor, MEK162, to exert a cytotoxic effect on GIST cell lines and the combination of phenothiazine with MEK162 induces apoptosis with diminished autophagy and the downregulation of ERK and downstream ELK1 and EGR1. As we observed paradoxical ERK activation following phenothiazine treatment, we then evaluated the possibility of combining phenothiazine with an MEK inhibitor to treat GISTs. As shown in Fig. 6, TFP or TDZ acted synergistically with the MEK inhibitor, MEK162, in inducing cytotoxic effects in the GIST48 and GIST882 cells.

The mechanisms of autophagy induction by phenothiazine are unclear. EGR1 is a member of the EGR family of $\mathrm{C} 2 \mathrm{H} 2$-type zinc finger proteins. It is a nuclear protein and functions as a transcriptional regulator, and it is also known to be responsible for environmental stress-induced autophagy, such as that caused by smoking (32). Additionally, EGR1 expression can be induced by ELK1, which is a member of the Ets family of transcription factors and of the ternary complex factor (TCF) subfamily, as well as a known nuclear target for the RAS/RAF/MAPK signaling cascade (33). Consequently, we considered that phenothiazine-induced autophagy may occur through ERK activation of the ELK1/EGR1 pathway. As shown in Fig. 7, TFP or TDZ induced autophagy with concomitant ERK, ELK1 and EGR1 activation. However, the combination of MEK162 and phenothiazine downregulated ERK, and downstream ELK1 and EGR1 with a resultant decrease in autophagy. Apoptosis, based on PARP cleavage, seemed more apparent in cells treated with the MEK162 and TFP combination. This result indicates that phenothiazine-induced autophagy may occur through the ERK/ELK1/EGR1 pathway, and the combination of phenothiazine with MEK inhibitor is a potential strategy for the treatment of GISTs.

\section{Discussion}

In this study, by uploading the differential expression gene sets of wild-type versus knockdown ETV1 to CMAP, we identified several agents with therapeutic potential. Among these, phenothiazine had not been reported previously, at least to the best of our knowledge. Phenothiazine-derived drugs were found to 
GIST882

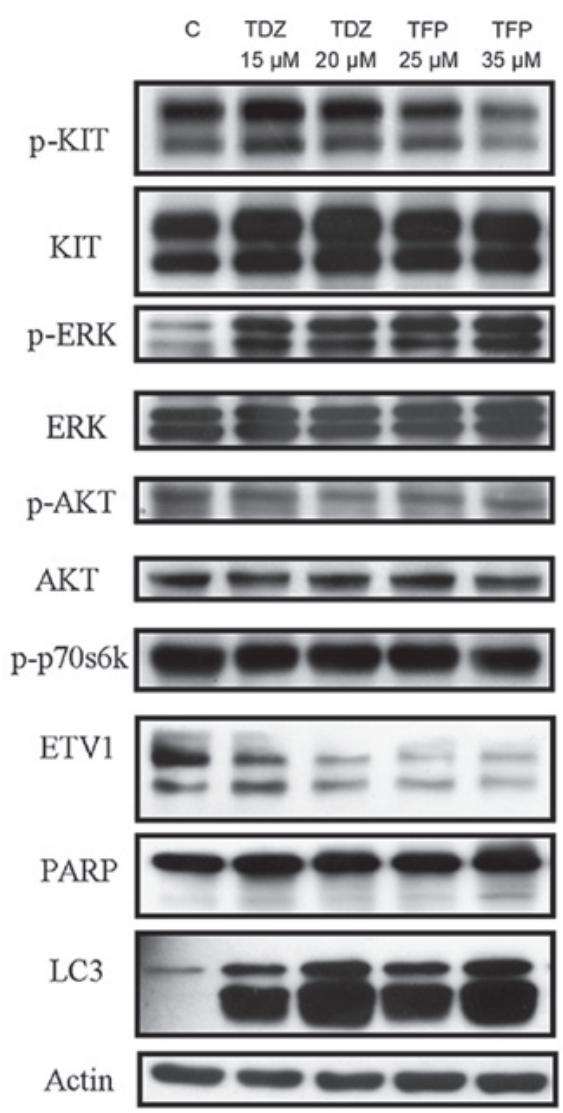

GIST48

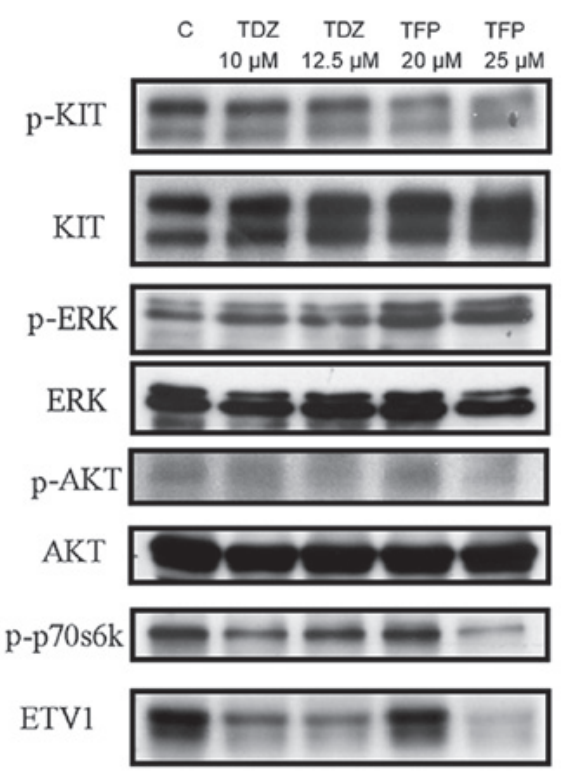

PARP

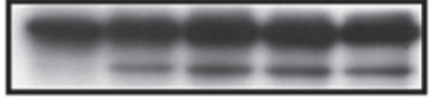

LC3

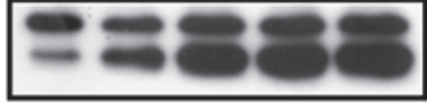

Actin

Figure 4. Western blot analysis of GIST882 (left panel) and GIST48 (right panel) before and after drug treatment. TFP and TDZ induced ETV1 downregulation with accompanying increased LC3-II expression. PARP cleavage was more prominent in GIST48. Paradoxical ERK activation was observed in both cell lines. Except with a relatively high concentration of TFP, no significant changes in KIT/AKT/mTOR (indicated by p-p70s6k) signaling were observed. TFP, trifluoperazine; TDZ, thioridazine.

A

GIST882
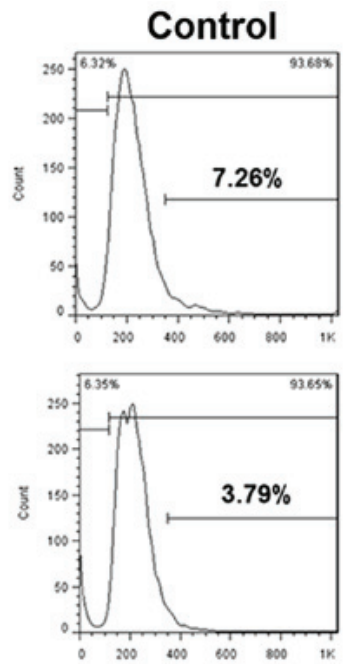

TFP
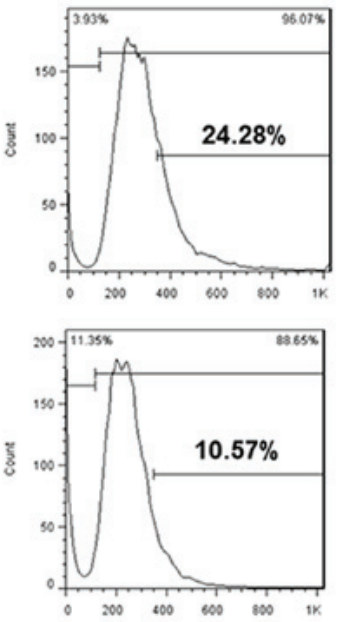

B

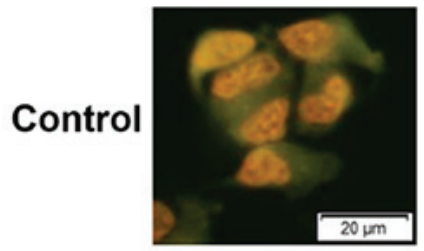

TFP

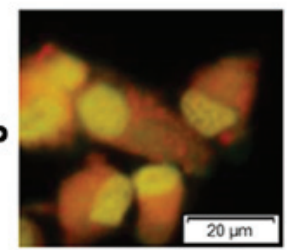

Figure 5. Quantitative FACS analysis of untreated and TFP-treated GIST cells stained with $10 \mu \mathrm{M}$ acridine orange (AO) (15 min). (A) Histograms of both cell lines (GIST882, $20 \mu \mathrm{M}, 72 \mathrm{~h}$; GIST48, $10 \mu \mathrm{M}, 72 \mathrm{~h}$ ) and (B) fluorescence microscopy of GIST48 show an increase of red fluorescence in the lysosomes (FL-3 or red dots by microscopy) following drug treatment (magnification, x200; scale bar, $20 \mu \mathrm{m}$ ). TFP, trifluoperazine; TDZ, thioridazine.

exert cytotoxicity and could induce apoptosis and autophagy in GISTs. Phenothiazine had little effect on the KIT/AKT/mTOR pathway, but instead paradoxically upregulated ERK activity. Phenothiazine may induce autophagy through the activation of the MEK/ERK/ELK1/EGR1 pathway, and the combination of phenothiazine and a MEK inhibitor had a synergistic cytotoxic effect on GISTs.

The important role of ETV1 in GIST was first revealed by Chi et al (20). Their study demonstrated that ETV1 was regulated by the KIT/MAP kinase pathway, and plays a key role in 

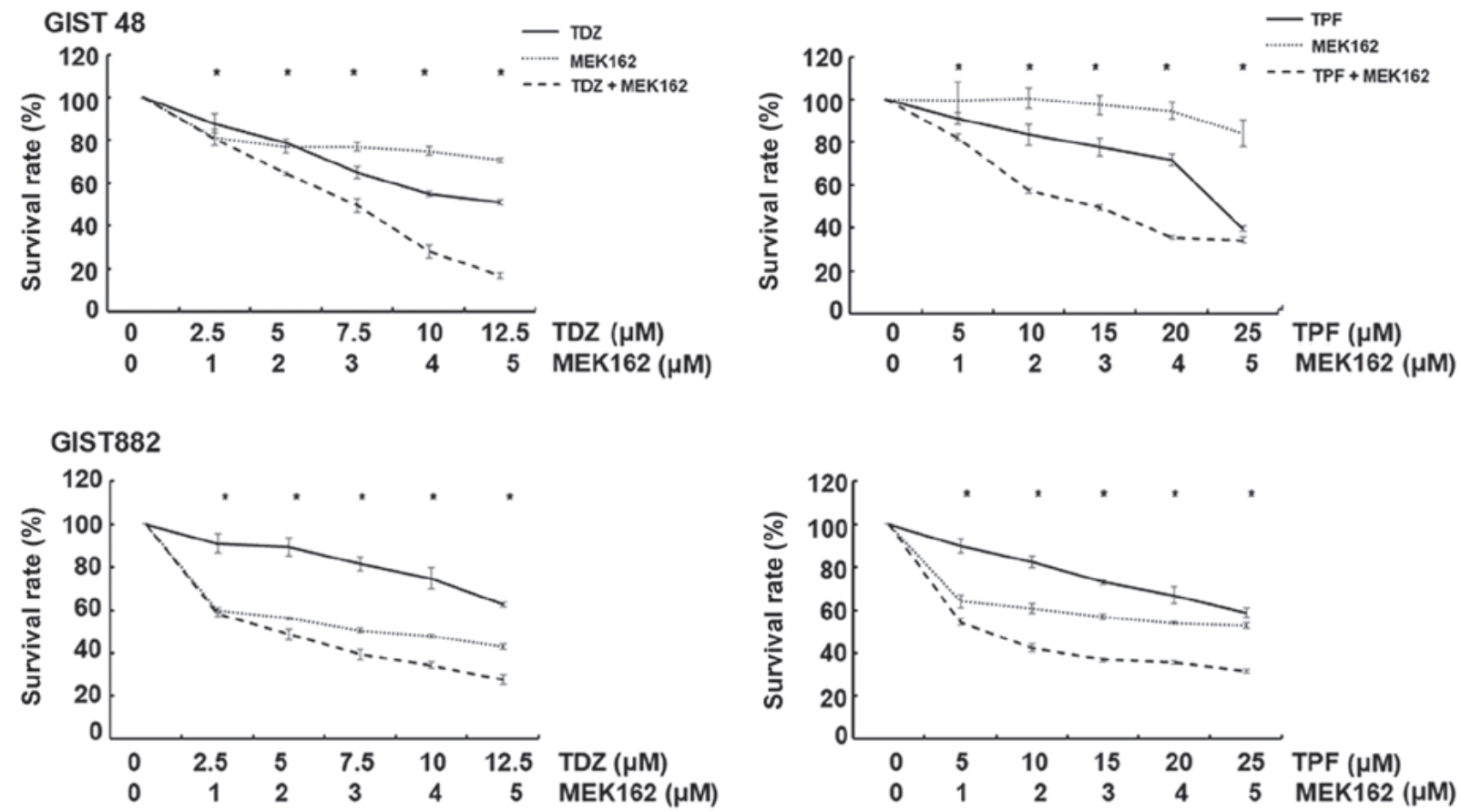

Figure 6. Viability of GIST48 and GIST882 cells following treatment with various concentrations of TDZ, TFP, or MEK162 alone, or a combination of phenothiazine with MEK162, was measured with the TACS ${ }^{\text {TM }}$ MTT cell proliferation assay (expressed as a percentage of viability under controlled culture conditions). A significant synergistic effect of combination of phenothiazine with MEK162 could be observed in both GIST cell lines at all levels of concentration [*, combination index $(\mathrm{CI})<1]$. TFP, trifluoperazine; TDZ, thioridazine.

A
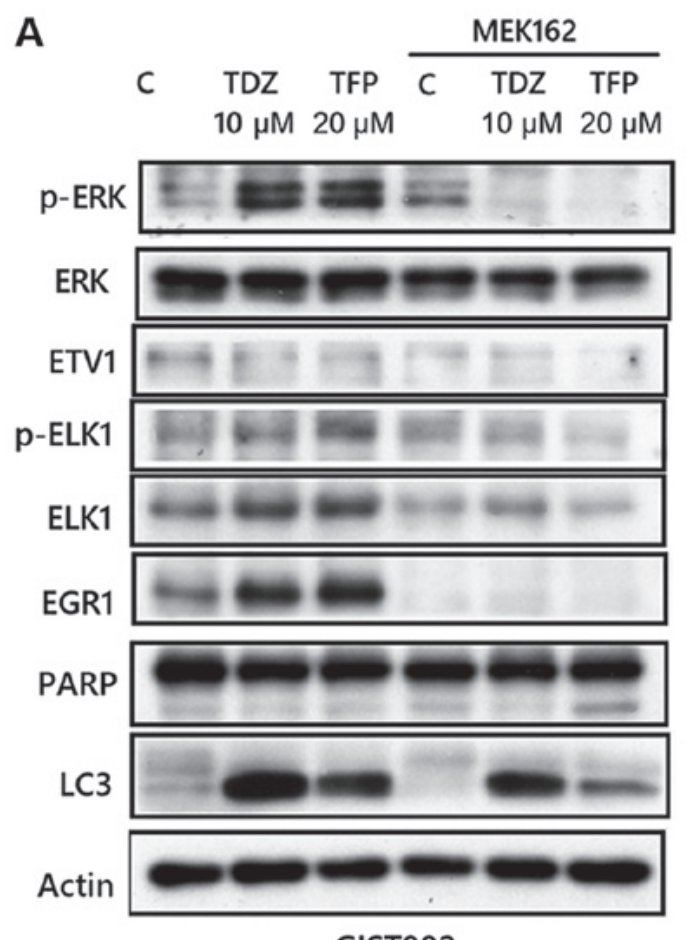

GIST882
B
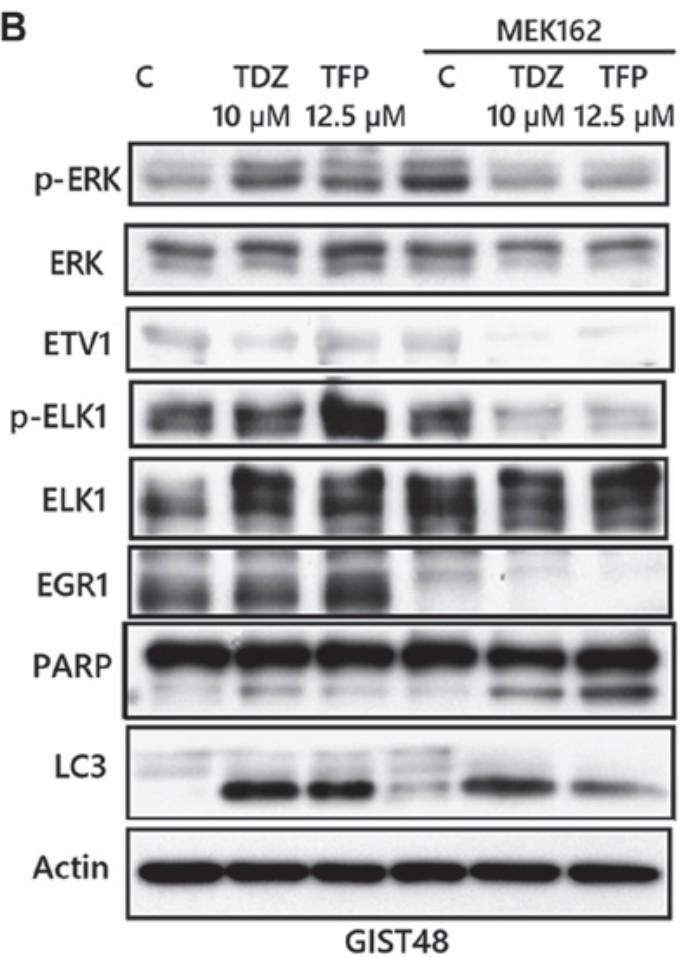

Figure 7. Western blot analysis of (A) GIST882 and (B) GIST48 cells following treatment with either TFP or TDZ, or in combination with MEK162. Increased LC3-II expression was found following TFP and TDZ treatment with concomitant activation of ERK, ELK1 and EGR1. Combination of MEK162 and phenothiazine diminished autophagy, enhanced apoptosis, and downregulate ERK and downstream ELK1 and EGR1.

an ICC-GIST-specific transcription network (20). Furthermore, a positive feedback circuit between the KIT/MAP kinase pathway and ETV1 was found, and a combination therapy of imatinib and MEK162 to target ETV1 stability resulted in considerable tumor suppressive effects (21). Their studies led us to explore the agents with ETV1-targeting potentials in GIST treatment.

In this study, through the analysis of a public-domain database, we found that ETV1, along with several known 
genes such as KIT, DOG1, and PKC $\theta$, was significantly overexpressed in GISTs in comparison with other sarcomas. This is consistent with previous reports $(20,34)$, again demonstrating the uniqueness and importance of ETV1 in GISTs. Subsequently, we uploaded an ETV1 knockout gene signature of GIST cell lines to CMAP, and we identified several ETV1 targeting agents. CMAP is a genome-wide transcriptional expression database generated from cultured human cells treated with bioactive small molecules. It applies a simple pattern-matching algorithm to discover the functional connections between drugs, genes, and diseases through common gene-expression changes. This database has been widely used in the discovery of novel agents and in deciphering previously unknown molecular mechanisms (35-38). In this study, through CMAP analysis of an ETV1 knockout gene signature of GIST cell lines, we discovered SAHA and trichostatin (two HDACIs) and TFP and TDZ (two drugs of phenothiazine class) as ETV1-targeting agents.

As expected, HDACIs were on the list, as a previous study has already shown their activity in downregulating KIT (14). In this study, we also demonstrated their activity by western blot analysis (Fig. 2). However, phenothiazine has not been previously reported to have anti-GIST activity, at least to the best of our knowledge. Phenothiazine has been identified as a class of potential anticancer drugs in previous publications. Possible mechanisms include inhibiting the PDK1/AKT pathway $(39,40)$, Wnt/b-catenin pathway (41), dopamine receptors (42), HSP70 (43) and DNA repair (44). In this study, we did not observe a significant effect of phenothiazine on the KIT/AKT/mTOR pathway. However, paradoxical ERK activation was found following treatment with the drugs.

The mechanisms of paradoxical ERK activation following phenothiazine or MEK162 treatment are not clear. ERK pathway activity is regulated by negative feedback at multiple levels, including the transcriptional activation of DUSP proteins that negatively regulate the pathway. ERK also phosphorylates and thus regulates upstream CRAF, SOS and MEK activity directly (45). One possible hypothesis is that these feedback inhibitory pathways may be temporarily shut down after phenothiazine- or MEK162- induced ERK inhibition, with resultant re-activation of ERK, and complete inhibition could only be achieved by combination of different agents targeting different levels. However, the specific underlying mechanisms warrant further investigation.

Although phenothiazine has been demonstrated to be a class of autophagy-inducing agents (31), the associated mechanism is still unclear. Chen et al discovered the role of EGR1 in autophagy by showing that cigarette smoke extract (CSE) could reduce HDAC activity, which resulted in increased binding of EGR1 and E2F factors to the LC3B promoter with subsequently increased LC3B expression in human pulmonary epithelial cells (32). Additionally, Guha et al revealed that lipopolysaccharides induced ELK1 phosphorylation through the MEK/ERK1/2 pathway with the subsequent induction of EGR1 expression in monocytes (33). As we observed ERK activation following phenothiazine treatment, we then explored the role of ERK activation in autophagy. In this study, we revealed that a combination of phenothiazine and a MEK inhibitor had a synergistic cytotoxic effect on GISTs. Western blot analysis indicated that MEK inhibition diminished autophagy and, together with phenothiazine, induced apoptosis. We also demonstrated that both ELK1 and EGR1 were activated/upregulated following TDZ and TFP treatment, but both were downregulated with a concomitant decrease in LC3-II expression after MEK inhibition. This result indicates that phenothiazine-induced autophagy may be mediated through the MEK/ERK/ELK1/EGR1 pathway and that the combination of phenothiazine with a MEK inhibitor may be a potential strategy for the treatment of GISTs.

In conclusion, in this study, by using bioinformatics analysis through CMAP, we identified TFP and TDZ, two drugs of phenothiazine class, as potential ETV1-targeting agents in GIST treatment. Phenothiazine-derived drugs induced apoptosis and autophagy in GISTs. Phenothiazine treatment was found to paradoxically upregulate ERK activity, but with little effect on the KIT/AKT/mTOR pathway. Phenothiazine may induce autophagy in GISTs through the MEK/ERK/ELK1/EGR1 pathway. A combination of phenothiazine and a MEK inhibitor exerted a synergistic cytotoxic effect on GISTs. Additional in vivo or clinical studies are warranted for further confirmation.

\section{Acknowledgements}

This manuscript was edited by Wallace Academic Editing.

\section{Funding}

This study was jointly supported by the grants from the Department of Health in Taiwan (Center of Excellence for Cancer Research at Taipei Veterans General Hospital, grant nos. DOH99-TD-C-111-007 and DOH100-TD-C-111-007, and the National Research Program for Biopharmaceuticals, grant no. DOH100-TD-PB-111-TM026), National Science Council (NSC 100-2314-B-075-081 and NSC 101-2314-B-075-029), Ministry of Science and Technology, Taiwan, (MOST 103-2314-B-075-066, MOST 105-2314-B-075-059, MOST 106-2314-B-075 -065), Taipei Veterans General Hospital (V102E8-003, V103E8-001, V101C-133, V102C-034,V103C-188, V104C-099, V104E16-001-MY3-1, V104E16-001-MY3-2, V104D16-001-MY3-3, V105C-094, V106C-160, V107C-085, V107D32-001-MY2-2, V108C-108) and from the Yen Tjing Ling Medical Foundation (grant no. CI-100-19, CI-103-6, CI-105-4) designated to CCY. This study was also supported by the Taiwan Clinical Oncology Research Foundation, and Chong Hin Loon Memorial Cancer and Biotherapy Research Center of National Yang-Ming University.

\section{Availability of data and materials}

The datasets used and/or analyzed during the current study are available from the corresponding author on reasonable request.

\section{Authors' contributions}

JAF, CCY, LTC and CFL were responsible for the design and conception of the study; WYC, YCL, CHY and YCC were responsible for data acquisition and interpretation; SCC, MHY and $\mathrm{YC}$ were responsible for the data analysis and drafting 
the work. All of the authors have read and approved the final manuscript for publication.

\section{Ethics approval and consent to participate}

Not applicable.

\section{Patient consent for publication}

Not applicable.

\section{Competing interests}

The authors declare that they have no competing interests.

\section{References}

1. Miettinen M and Lasota J: Gastrointestinal stromal tumors: Review on morphology, molecular pathology, prognosis, and differential diagnosis. Arch Pathol Lab Med 130: 1466-1478, 2006.

2. Reith JD, Goldblum JR, Lyles RH and Weiss SW: Extragastrointestinal (soft tissue) stromal tumors: An analysis of 48 cases with emphasis on histologic predictors of outcome. Mod Pathol 13: 577-585, 2000.

3. Tran T, Davila JA and El-Serag HB: The epidemiology of malignant gastrointestinal stromal tumors: An analysis of 1,458 cases from 1992 to 2000. Am J Gastroenterol 100: 162-168, 2005.

4. Hirota S, Isozaki K, Moriyama Y, Hashimoto K, Nishida T, Ishiguro S, Kawano K, Hanada M, Kurata A, Takeda M, et al: Gain-of-function mutations of c-kit in human gastrointestinal stromal tumors. Science 279: 577-580, 1998.

5. Heinrich MC, Corless CL, Duensing A, McGreevey L, Chen CJ, Joseph N, Singer S, Griffith DJ, Haley A, Town A, et al: PDGFRA activating mutations in gastrointestinal stromal tumors. Science 299: 708-710, 2003

6. Blanke CD, Demetri GD, von Mehren M, Heinrich MC, Eisenberg B, Fletcher JA, Corless CL, Fletcher CD, Roberts PJ, Heinz D, et al: Long-term results from a randomized phase II trial of standard- versus higher-dose imatinib mesylate for patients with unresectable or metastatic gastrointestinal stromal tumors expressing KIT. J Clin Oncol 26: 620-625, 2008.

7. Yeh CN, Chen YY, Tseng JH, Chen JS, Chen TW, Tsai CY, Cheng CT, Jan YY and Chen MF: Imatinib mesylate forpatients with recurrent or metastatic gastrointestinal stromal tumors expressing KIT: A decade experience from Taiwan. Trans Oncol 4: 328-335, 2011.

8. Heinrich MC, Corless CL, Blanke CD, Demetri GD, Joensuu H, Roberts PJ, Eisenberg BL, von Mehren M, Fletcher CD, Sandau K, et al: Molecular correlates of imatinib resistance in gastrointestinal stromal tumors. J Clin Oncol 24: 4764-4774, 2006.

9. Heinrich MC, Maki RG, Corless CL, Antonescu CR, Harlow A, Griffith D, Town A, McKinley A, Ou WB, Fletcher JA, et al: Primary and secondary kinase genotypes correlate with the biological and clinical activity of sunitinib in imatinib-resistant gastrointestinal stromal tumor. J Clin Oncol 26: 5352-5359, 2008

10. Demetri GD, van Oosterom AT, Garrett CR, Blackstein ME, Shah MH, Verweij J, McArthur G, Judson IR, Heinrich MC, Morgan JA, et al: Efficacy and safety of sunitinib in patients with advanced gastrointestinal stromal tumour after failure of imatinib: A randomised controlled trial. Lancet 368: 1329-1338, 2006.

11. Demetri GD, Reichardt P, Kang YK, Blay JY, Rutkowski P, Gelderblom H, Hohenberger P, Leahy M, von Mehren M, Joensuu H, et al; GRID study investigators: Efficacy and safety of regorafenib for advanced gastrointestinal stromal tumours after failure of imatinib and sunitinib (GRID): An international, multicentre, randomised, placebo-controlled, phase 3 trial. Lancet 381: 295-302, 2013.

12. Yeh CN, Chen MH, Chen YY, Yang CY, Yen CC, Tzen CY, Chen LT and Chen JS: A phase II trial of regorafenib in patients with metastatic and/or a unresectable gastrointestinal stromal tumor harboring secondary mutations of exon 17. Oncotarget 8: 44121-44130, 2017.
13. Bauer S, Yu LK, Demetri GD and Fletcher JA: Heat shock protein 90 inhibition in imatinib-resistant gastrointestinal stromal tumor. Cancer Res 66: 9153-9161, 2006.

14. Mühlenberg T, Zhang Y, Wagner AJ, Grabellus F, Bradner J, Taeger G, Lang H, Taguchi T, Schuler M, Fletcher JA, et al: Inhibitors of deacetylases suppress oncogenic KIT signaling, acetylate HSP90, and induce apoptosis in gastrointestinal stromal tumors. Cancer Res 69: 6941-6950, 2009.

15. Bauer S, Duensing A, Demetri GD and Fletcher JA: KIT oncogenic signaling mechanisms in imatinib-resistant gastrointestinal stromal tumor: PI3-kinase/AKT is a crucial survival pathway. Oncogene 26: 7560-7568, 2007.

16. Pantaleo MA, Nicoletti G, Nanni C, Gnocchi C, Landuzzi L, Quarta C, Boschi S, Nannini M, Di Battista M, Castellucci P, et al: Preclinical evaluation of KIT/PDGFRA and mTOR inhibitors in gastrointestinal stromal tumors using small animal FDG PET. J Exp Clin Cancer Res 29: 173, 2010.

17. Bendell JC, Bauer TM, Lamar R, Joseph M, Penley W, Thompson DS, Spigel DR, Owera R, Lane CM, Earwood C, et al: A phase 2 study of the Hsp90 inhibitor AUY922 as treatment for patients with refractory gastrointestinal stromal tumors. Cancer Invest 34: 265-270, 2016.

18. Deming DA, Ninan J, Bailey HH, Kolesar JM, Eickhoff J, Reid JM, Ames MM, McGovern RM, Alberti D, Marnocha R, et al: A Phase I study of intermittently dosed vorinostat in combination with bortezomib in patients with advanced solid tumors. Invest New Drugs 32: 323-329, 2014.

19. Schöffski P, Reichardt P, Blay JY, Dumez H, Morgan JA, Ray-Coquard I, Hollaender N, Jappe A and Demetri GD: A phase I-II study of everolimus (RAD001) in combination with imatinib in patients with imatinib-resistant gastrointestinal stromal tumors. Ann Oncol 21: 1990-1998, 2010.

20. Chi P, Chen Y, Zhang L, Guo X, Wongvipat J, Shamu T, Fletcher JA, Dewell S, Maki RG, Zheng D, et al: ETV1 is a lineage survival factor that cooperates with KIT in gastrointestinal stromal tumours. Nature 467: 849-853, 2010.

21. Ran L, Sirota I, Cao Z, Murphy D, Chen Y, Shukla S, Xie Y, Kaufmann MC, Gao D, Zhu S, et al: Combined inhibition of MAP kinase and KIT signaling synergistically destabilizes ETV1 and suppresses GIST tumor growth. Cancer Discov 5: 304-315, 2015.

22. Lamb J, Crawford ED, Peck D, Modell JW, Blat IC, Wrobel MJ, Lerner J, Brunet JP, Subramanian A, Ross KN, et al: The Connectivity Map: Using gene-expression signatures to connect small molecules, genes, and disease. Science 313: 1929-1935, 2006.

23. Lamb J: The Connectivity Map: A new tool for biomedical research. Nat Rev Cancer 7: 54-60, 2007.

24. Li C and Hung WW: Model-based analysis of oligonucleotide arrays: model validation, design issues and standard error application. Genome Biol 2: RESEARCH0032, 2001.

25. Li C and Wong WH: Model-based analysis of oligonucleotide arrays: Expression index computation and outlier detection. Proc Natl Acad Sci USA 98: 31-36, 2001.

26. Tuveson DA, Willis NA, Jacks T, Griffin JD, Singer S, Fletcher CD, Fletcher JA and Demetri GD: STI571 inactivation of the gastrointestinal stromal tumor c-KIT oncoprotein: Biological and clinical implications. Oncogene 20: 5054-5058, 2001.

27. Sun X, Hung K, Wu L, Sidransky D and Guo B: Detection of tumor mutations in the presence of excess amounts of normal DNA. Nat Biotechnol 20: 186-189, 2002.

28. Ross P, Hall L, Smirnov I and Haff L: High level multiplex genotyping by MALDI-TOF mass spectrometry. Nat Biotechnol 16: 1347-1351, 1998.

29. Mosmann T: Rapid colorimetric assay for cellular growth and survival: Application to proliferation and cytotoxicity assays. J Immunol Methods 65: 55-63, 1983.

30. Chou TC and Talalay P: Quantitative analysis of dose-effect relationships: The combined effects of multiple drugs or enzyme inhibitors. Adv Enzyme Regul 22: 27-55, 1984

31. Zhang L, Yu J, Pan H, Hu P, Hao Y, Cai W, Zhu H, Yu AD, Xie X, Ma D, et al: Small molecule regulators of autophagy identified by an image-based high-throughput screen. Proc Natl Acad Sci USA 104: 19023-19028, 2007.

32. Chen ZH, Kim HP, Sciurba FC, Lee SJ, Feghali-Bostwick C, Stolz DB, Dhir R, Landreneau RJ, Schuchert MJ, Yousem SA, et al: Egr-1 regulates autophagy in cigarette smoke-induced chronic obstructive pulmonary disease. PLoS One 3: e3316, 2008. 
33. Guha M, O'Connell MA, Pawlinski R, Hollis A, McGovern P, Yan SF, Stern D and Mackman N: Lipopolysaccharide activation of the MEK-ERK1/2 pathway in human monocytic cells mediates tissue factor and tumor necrosis factor alpha expression by inducing Elk-1 phosphorylation and Egr-1 expression. Blood 98: 1429-1439, 2001

34. Jang BG, Lee HE and Kim WH: ETV1 mRNA is specifically expressed in gastrointestinal stromal tumors. Virchows Arch 467: 393-403, 2015.

35. Wang SE, Xiang B, Guix M, Olivares MG, Parker J, Chung $\mathrm{CH}$ Pandiella A and Arteaga CL: Transforming growth factor beta engages TACE and ErbB3 to activate phosphatidylinositol-3 kinase/Akt in ErbB2-overexpressing breast cancer and desensitizes cells to trastuzumab. Mol Cell Biol 28: 5605-5620, 2008.

36. Hieronymus H, Lamb J, Ross KN, Peng XP, Clement C, Rodina A, Nieto M, Du J, Stegmaier K, Raj SM, et al: Gene expression signature-based chemical genomic prediction identifies a novel class of HSP90 pathway modulators. Cancer Cell 10: 321-330, 2006.

37. Wei G, Twomey D, Lamb J, Schlis K, Agarwal J, Stam RW, Opferman JT, Sallan SE, den Boer ML, Pieters R, et al: Gene expression-based chemical genomics identifies rapamycin as a modulator of MCL1 and glucocorticoid resistance. Cancer Cell 10: 331-342, 2006.

38. Garman KS, Acharya CR, Edelman E, Grade M, Gaedcke J, Sud S, Barry W, Diehl AM, Provenzale D, Ginsburg GS, et al: A genomic approach to colon cancer risk stratification yields biologic insights into therapeutic opportunities. Proc Natl Acad Sci USA 105: 19432-19437, 2008.

39. Choi JH, Yang YR, Lee SK, Kim SH, Kim YH, Cha JY, Oh SW, Ha JR, Ryu SH and Suh PG: Potential inhibition of PDK1/Akt signaling by phenothiazines suppresses cancer cell proliferation and survival. Ann NY Acad Sci 1138: 393-403, 2008.
40. Rho SB, Kim BR and Kang S: A gene signature-based approach identifies thioridazine as an inhibitor of phosphatidylinositol-3'-kinase (PI3K)/AKT pathway in ovarian cancer cells. Gynecol Oncol 120: 121-127, 2011.

41. Yeh CT, Wu AT, Chang PM, Chen KY, Yang CN, Yang SC, Ho CC, Chen CC, Kuo YL, Lee PY, et al: Trifluoperazine, an antipsychotic agent, inhibits cancer stem cell growth and overcomes drug resistance of lung cancer. Am J Respir Crit Care Med 186: 1180-1188, 2012.

42. Sachlos E, Risueño RM, Laronde S, Shapovalova Z, Lee JH, Russell J, Malig M, McNicol JD, Fiebig-Comyn A, Graham M, et al: Identification of drugs including a dopamine receptor antagonist that selectively target cancer stem cells. Cell 149: 1284-1297, 2012.

43. Koren J III, Jinwal UK, Jin Y, O'Leary J, Jones JR, Johnson AG, Blair LJ, Abisambra JF, Chang L, Miyata Y, et al: Facilitating Akt clearance via manipulation of Hsp70 activity and levels. J Biol Chem 285: 2498-2505, 2010.

44. Polischouk AG, Holgersson A, Zong D, Stenerlöw B, Karlsson HL, Möller L, Viktorsson K and Lewensohn R: The antipsychotic drug trifluoperazine inhibits DNA repair and sensitizes non small cell lung carcinoma cells to DNA double-strand break induced cell death. Mol Cancer Ther 6: 2303-2309, 2007.

45. Liu F, Yang X, Geng $M$ and Huang M: Targeting ERK, an Achilles' Heel of the MAPK pathway, in cancer therapy. Acta Pharm Sin B 8: 552-562, 2018. 